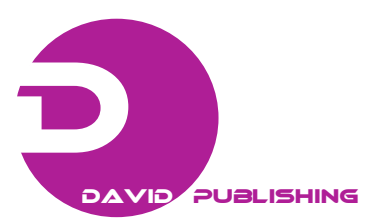

\title{
A Game Theory Approach Using Excel
}

\author{
Giancarlo de França Aguiar ${ }^{1}$, Alessandro Brawerman ${ }^{2,3}$, Barbara C. X. C. Aguiar ${ }^{4}$ and Volmir E. Wilhelm ${ }^{4}$ \\ 1. Department of Mathematics, Federal Institute of Paraná, Curitiba 82530-230, Brazil \\ 2. Department of Computer Engineering, Positivo University, Curitiba 81280-330, Brazil \\ 3. Sector of Professional and Technological Education, Federal University of Paraná, Curitiba 81520-260, Brazil \\ 4. Department of Mathematics, Federal University of Paraná, Curitiba 80060-000, Brazil
}

Received: June 24, 2014 / Accepted: July 08, 2014 / Published: September 25, 2014.

\begin{abstract}
Understanding the behavior of companies in market conditions has been an important topic of studies for the economists and mathematicians. Many companies have treated their relationship of service providers as a game. Thus, the attitudes of the players can be endowed with rational thinking, which leads to the conclusion that the use of game theory as a tool to understand such behavior is of great importance. Based on the previous studies, this paper presents a study on the cooperative game theory, discussing the Nash equilibrium in pure and mixed strategies, treating solutions using the minimax John Von Neumann theorem and illustrating a mathematical modeling of a game theory problem. Furthermore, a solution of linear programming using Microsoft Excel is also proposed and presented. The methodology adopted to model the problem may help students to familiarize themselves to game theory.
\end{abstract}

Key words: Cooperative game theory, Nash equilibrium, linear programming, Microsoft Excel.

\section{Introduction}

Understanding the behavior of companies in market conditions has been an important topic of studies for the economists and mathematicians. According to Ref. [1], the impact of decisions in organizations is interdependent and has been widely discussed in the microeconomic literature since the 19th century.

Currently, many companies have treated their relationship of service providers as a game, and the game, as such, takes into account the attitudes of their players who aim to maximize their interests. According to Ref. [2], the game has achieved greater prominence as a methodology and should prepare players to make their companies competitive in relation to the market.

In turn, as the attitudes of the players can be endowed with rational thinking, we conclude that the game is about strategies, which leads us to moderate the use of game theory as a tool to understand such

Corresponding author: Giancarlo de França Aguiar, M.Sc., assistant professor, research fields: game theory, DEA. E-mail: giancarlo.aguiar@ifpr.edu.br. behavior.

Game theory has become of great importance for the modern economic thinking. It can be considered as a methodology and has been used in various fields of science, such as biology [3], engineering [4], economics $[5,6]$, politics $[7,8]$ and among others, in order to organize mathematically possible decisions of players in the middle of a game.

Based on those studies, this work weaves a theoretical basis on the Theory of Cooperative Games, illustrating the Nash equilibrium in pure and mixed strategies, addressing solutions to a game using the minimax and maximin theorems of John Von Neumann. Finally, a mathematical model (linear programming) of a problem with our solution is modeled in a Microsoft Excel spreadsheet.

The paper is organized as follows: Section 2 presents a brief overview of fundamental theory; Section 3 discusses a mathematical modeling of our proposed game and presents a model in an Excel spreadsheet; and Section 4 gives conclusions and presents future work. 


\section{Fundamental Theory}

This section presents some fundamental aspects and a theory that the authors consider important to offer a better understanding of this work. We discuss the Nash equilibrium and the minimax theorem.

\subsection{Strategic Solution or Nash Equilibrium}

A strategic solution to a game or Nash equilibrium is a point (or several points), where each player has no incentive (since the objective in the game is for each player to maximize their rewards) to change its strategy if the other players also do not.

The definition of Nash equilibrium: we say that a strategy profile

$$
s^{*}=\left(s_{1}^{*}, \ldots, s_{(i-1)}^{*}, s_{i}^{*}, s_{(i+1)}^{*}, \ldots, s_{n}^{*}\right) \in S
$$

is a Nash equilibrium if

$$
u_{i}\left(s_{i}^{*}, s_{-i}^{*}\right) \geq u_{i}\left(s_{i j_{i}}, s_{-i}^{*}\right)
$$

$\forall i=1, \ldots, n$ and $\forall j_{i}=1, \ldots, m_{i}$ with $m_{i} \geq 2$,

where, $u_{i}$ is the utility function or payoff of a player $g_{i}, s_{i}$ is a given strategy of a player $g_{i}, s_{-i}$ is a given strategy of some other players (with exception of $g_{i}$ ) and the symbol $(*)$ represents that the strategy is a Nash equilibrium.

A strategy $s_{i}^{*}$ of a player $g_{i}$ is considered to be the best move to a particular strategy $s_{-i}$ of another player, if there is no other strategy available for the player $g_{i}$ to provide him/her a better reward to the chosen strategy. This concept should be extended to all players, i.e., the Nash equilibrium is a solution to the game in which all strategies taken by all players are always the best strategic solutions to the strategies adopted by other players.

Among the classical problems in the literature on game theory, the battle of the sexes $[9,10]$ is a fictional situation in which a couple has to decide on the program they will participate. The problem describes three options: go to a football match, go to the theater or not to go anywhere. This problem is reported as a problem of pure strategies.

When a player $g_{i}$ decides alternately to choose a strategy $s_{i}$ among all its other strategies, assigning each a weight (probability) and not deciding any strategy by choosing one (pure), we say that the player is using a mixed strategy. All elementary considerations used for games with pure strategies can be extended to mixed strategies.

According to Ref. [11], a mixed strategy $p_{i}$ for the player $g_{i} \in G$ is a probability distribution over the set $S_{i}$ of pure strategies of the player, i.e., $p_{i}$ is a member of the set

$$
\Delta_{m_{i}}=\left\{\left(x_{1}, x_{2}, \ldots, x_{m_{i}}\right) \in \mathbb{R}^{m_{i}} \mid x_{1} \geq 0, \ldots, x_{m_{i}} \geq 0 \text { and } \sum_{k=1}^{m_{i}} x_{k=1}\right\}
$$

Thus, if $p_{i}=\left(p_{i 1}, p_{i 2}, \ldots, p_{i m_{i}}\right)$, then,

$$
p_{i 1} \geq 0, p_{i 2} \geq 0, \ldots, p_{i m_{i}} \geq 0 \text { and } \sum_{k=1}^{m_{i}} p_{i k}=1
$$

The space of all mixed strategy profiles is the Cartesian product, $\Delta=\Delta_{m_{1}} x \Delta_{m_{2}} x \ldots x \Delta_{m_{n}}$, and this space is called a mixed strategy. A vector $p \in \Delta$ is called a profile of mixed strategy. As in pure strategies, we will use the notation $p_{-i}$ to represent the strategies of all players except the player $g_{i}$ himself. Each mixed strategy profile $p=\left(p_{1}, p_{2}, \ldots, p_{n}\right) \in \Delta$ determines an expected payoff of a weighted average of the payoffs, by probability distributions $p_{1}, \ldots, p_{n}$.

More precisely, if

$$
\begin{gathered}
p=\left(p_{1} ; p_{2} ; \ldots ; p_{n}\right) \\
p=\left(\begin{array}{c}
p_{11}, p_{12}, \ldots, p_{1 m_{1}} ; p_{21}, p_{22}, \ldots, \\
p_{2 m_{2}} ; \ldots ; p_{n 1}, p_{n 2}, \ldots, p_{n m_{n}}
\end{array}\right)
\end{gathered}
$$

then,

$$
\begin{aligned}
& u_{i}(p) \\
& =\sum_{j_{1}=1}^{m_{1}} \sum_{j_{2}=1}^{m_{2}} \ldots \sum_{j_{n}=1}^{m_{n}}\left(\prod_{k=1}^{n} p_{k j_{k}} u_{i}\left(s_{1 j_{1}}, s_{2 j_{2}}, \ldots, s_{n j_{n}}\right)\right)
\end{aligned}
$$

A set of mixed strategies much discussed in the literature is the game of choice between face and crown coins, known as matching pennies, which can be studied in Refs. [9, 12].

\subsection{Minimax Theorem and Mixed Strategies}

It follows below the John Von Neumann minimax theorem for problems with mixed strategies. 
Minimax theorem: if mixed strategies are allowed, the pair of mixed strategies which is great according to the minimax criterion provides a stable solution $\underline{v}=\bar{v}=v$, so that no player can improve their situation by changing its strategy.

Theorem: a strategy profile $\left(p^{*}, q^{*}\right)$ is a Nash equilibrium of a two-player game with constant sum defined by the payoff matrix of the row player $A=$ $\left(a_{i j}\right)_{m \times n}$ if and only if

$$
\max _{1 \leq k \leq m} \min _{1 \leq l \leq n} a_{k l}=\min _{1 \leq l \leq n} \max _{1 \leq k \leq m} a_{k l}=p^{* T} A q^{*}
$$

Theorem (Von Neumann minimax): for every zero-sum game with two players represented by the payoff matrix of the row player, there is always a mixed strategy profile $\left(p^{*}, q^{*}\right) \in \Delta_{m} x \Delta_{n}$ satisfying

$$
\begin{aligned}
v_{l}(A)=\max _{p \in \Delta_{m}} \min _{q \in \Delta_{n}} p^{T} A q=p^{* t} A q^{*} \\
=\min _{q \in \Delta_{n}} \max _{p \in \Delta_{m}} p^{T} A q=v_{c}(A)
\end{aligned}
$$

In particular, it is a Nash equilibrium of the game [13].

\section{Results and Discussion}

We first begin by presenting/modeling a game of two players (politicians), each with three strategies. The players payoff matrix is shown in Table 1.

Considering the game is between two candidates for mayor of a given city, it is fundamental that they plan ahead the last two days of the campaign. Politicians intend to campaign in two different neighborhoods (B and FL). Each candidate may perform three strategies: campaign two days in B, campaign two days in FL or campaign one day in each neighborhood.

With a brief analysis, it is concluded that this game does not have a minimax solution in pure strategies because it is a game with no saddle point. According to Table 1 , selecting the maximum result from line one (2), the maximum result from line two (5) and the maximum from line three (3), the minimum of the maximum is equal to 2 , thus, $\operatorname{minimax}=2$. Now,

\begin{tabular}{|c|c|c|c|c|}
\hline & & \multicolumn{3}{|c|}{ Politician 2} \\
\hline & & $\begin{array}{l}\text { One day in each } \\
\text { neighborhood }\end{array}$ & Two days in B & Two days in FL \\
\hline \multirow[t]{3}{*}{ Politician 1} & $\begin{array}{l}\text { One day in each } \\
\text { neighborhood }\end{array}$ & 0 & -2 & 2 \\
\hline & Two days in B & 5 & 4 & -3 \\
\hline & Two days in FL & 2 & 3 & -4 \\
\hline
\end{tabular}
selecting the minimum result of column one (0), the
Table 1 The game of political campaign.

minimum result of column two (-2) and the minimum value from column three (-4), the maximum of the minimum is equal to 0 , thus, $\operatorname{maximin}=0$. Since Minimax $\neq$ Maxmin, then the game does not have a solution in pure strategies. Let us try a linear programming approach to find the solution to this problem.

According to Ref. [14], the linear programming problems are business situations (problems of production, resource allocation, workforce planning and among others) that can be described/modeled mathematically in an organized manner through functions and equations, and thus, with their solutions simulate real situations and find alternatives with the needs of organizations. So the problem of player A can be written as:

$\operatorname{Max} Z=v$

such as:

$$
\begin{gathered}
v-0 x_{1}-5 x_{2}-2 x_{3} \leq 0 \\
v+2 x_{1}-4 x_{2}-3 x_{3} \leq 0 \\
v-2 x_{1}+3 x_{2}+4 x_{3} \leq 0 \\
x_{1}+x_{2}+x_{3}=1 \\
x_{1}, x_{2}, x_{3} \geq 0 \\
v \text { free }
\end{gathered}
$$

The previous model can be solved using a linear programming software, however, this time, we will use an electronic Microsoft Excel tool to assist in the modeling and resolution (proposal of this paper).

Fig. 1 illustrates the data entered in a Microsoft Excel spreadsheet. The only cells that have formulas are:

- D3 cells (= G4), which is the objective function of the cell and receives the value of the variable " $v$ ";

- J9 (=D9*G4+E9*H4+F9*I4+G9*J4), which is the restriction of one model;

- J10 (=D10*G4+E10*H4+F10*I4+G10*J4), which is the second constraint model; 


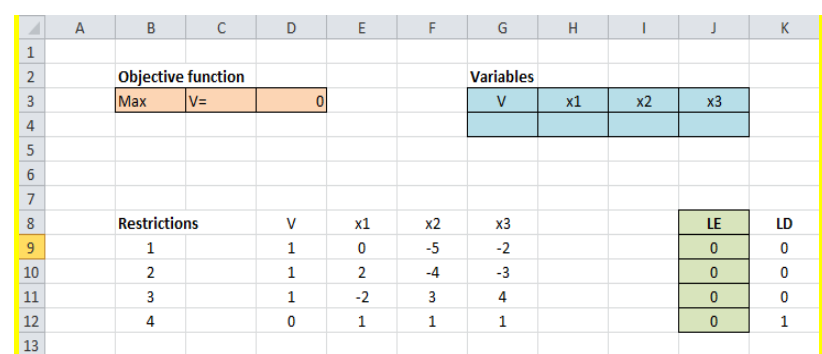

Fig. 1 Modeling in Excel.

- J11 (=D11*G4+E11*H4+F11*I4+G11*J4), which is the third constraint model;

$$
\text { - J12 (=D12*G4+E12*H4+F12*I4+G12*J4), }
$$

which is the fourth constraint model.

Following, one can click in the Microsoft Excel tool/data tab, and then tool/solver tab (if the tab solver exists, the user must customize his toolbar from the main menu, looking for the commands tab, then supplements and clicking solver). Fig. 2 illustrates the new window that pops up. The user must define the purpose by clicking in cell D3, then set up if the problem is the maximum or minimum (maximum in this case). When changing the variable cells, the user must place the cells of the variables (\$G\$4:\$J4), and then click add. A new window will pop up and the user must click on cell reference in cell (J9), the inequality (<=) is already correct and in the restriction tab, the user must click in cell K9. Click ok and perform the previous procedure to other restrictions.

Finally, one can click on the solve button and then ok. The solution will appear as shown in Fig. 3.

The spreadsheet shows the following solution for the player

$\left(v, x_{1}, x_{2}, x_{3}\right) \cong(0.1818 ; 0.6363 ; 0.3636 ; 0) \cong$ $\left(\frac{2}{11}, \frac{7}{11}, \frac{4}{11}, 0\right)$, i.e., player A should use the strategy seven times in every 11 attempts, the strategy four times two in every 11 attempts and discard the third strategy, getting the payoff $\max v=\frac{2}{11}$. The solution for player B may be constructed analogously.

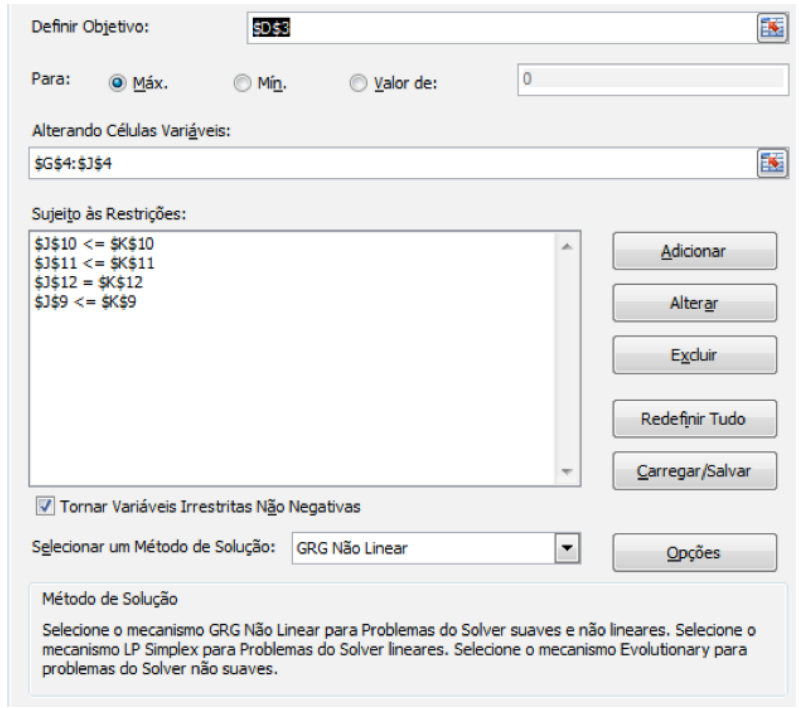

Fig. 2 Tools solver.

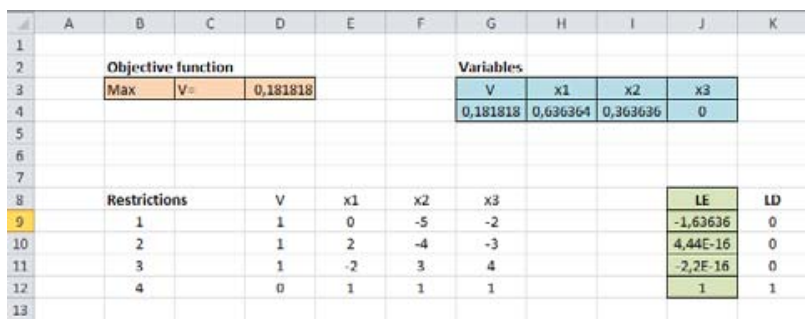

Fig. 3 Solution for the game.

\section{Conclusions}

This work showed a theoretical treatment for beginners who aim to know game theory, illustrating a game and a discussion of its solution (Nash equilibrium for pure strategies) and a game and a discussion of its solution (Nash equilibrium for mixed strategies).

According to Ref. [15], in game theory, if there are marked differences between the Nash equilibrium and the predicted results, then probably there was some sort of misunderstanding on the part of players (decision makers), or one of the players may not have understood clearly the priorities of his opponent, or one of them may not have understood the game or not being rational in their decisions.

This work also illustrated the minimax theorem of John von Neumann and an example problem with two players and three strategies. Here we show how to formalize the mathematical modeling of the problem 
and how to build a step-by-step modeling using a Microsoft Excel spreadsheet.

As future work we intend to implement a more generic game that may be accessed over the Internet.

\section{References}

[1] Leeflang, P. S. H. 2008. "Modeling Competitive Reaction Effects.” Schmalenbach Business Review 60: 322-58.

[2] Orti, P. S., Rodrigues, J. de S., and Albino, J. P. 2008. “Critical Success Factors in Business Games.” Presented at XV SIMPEP, UNESP, Bauru.

[3] Gatenby, R. A., and Vincent, T. L. 2003. “Application of Quantitative Models from Population Biology and Evolutionary Game Theory to Tumor Therapeutic Strategies.” Molecular Cancer Therapeutics 2: 919-27.

[4] Peldschus, F. 2008. "Experience of the Game Theory Application in Construction Management." Technological and Economic Development of Economy 14 (4): 531-45.

[5] Rabin, M. 1993. "Incorporating Fairness into Game Theory and Economics." The American Economic Review 91 (4): 1180-3.

[6] Roth, A. E. 2002. "The Economist as Engineer: Game Theory, Experimentation, and Computation as Tools for Design Economics.” Econometrica 70 (4): 1341-78.

[7] Munck, G. L. 2000. "Game Theory and Comparative Politics: New Perspectives, Old Interests.” DATA-Journal of Social Science 43 (3): 559-600.

[8] Mesquita, B. B. 2006. "The Evolution of Political Science.” American Political Science Review 100 (4): 637-42.

[9] Fiani, R. 2009. Game Theory with Applications in Economics, Management and Social Sciences. 3rd ed. Rio de Janeiro: Elsevier.

[10] Camerer, C. F. 2011. Behavioral Game Theory-Experiments in Strategic Interaction. USA: Princeton University Press.

[11] SARTINI et al., "An Introduction to Game Theory”. II Biennial SBM Federal University of Bahia, Oct. 25-29, 2004.

[12] Goeree, J. K., Holt, C. A., and Palfrey, T. R. 2003. "Risk Averse Behavior in Generalized Matching Pennies Games." Games and Economic Behavior 45 (1): 97-113.

[13] Oliveira, F. A., and Araújo, M. A. 2010. "The Minimax Theorem of von Neumann.” Presented at the XXXIII CNMAC, Montpellier, France.

[14] Aguiar, G. de F., Aguiar, B. de C. X. C., and Wilhelm, V. E. 2006. "Obtaining Efficiency Ratios Methodology for Data Envelopment Analysis Using Microsoft Excel spreadsheet.” DaVinci Magazine 3 (1): 157-70.

[15] Silveira, L. T., and Burnquist, H. L. 2009. "Procedure for Decision Analysis for the Prevention of Diseases in Animals: An Application of Game Theory.” Journal of Economics and Rural Sociology 47 (2): 435-64. 